\title{
Association of single-nucleotide polymorphisms in the STAT3 gene with autoimmune thyroid disease in Chinese individuals
}

\author{
Ling Xiao - Fatuma-Said Muhali • Tian-tian Cai • Rong-hua Song • Renming Hu • \\ Xiao-hong Shi • Wen-juan Jiang • Dan-Feng Li • Shuang-tao He • Jian Xu • \\ Jin-an Zhang
}

Received: 20 May 2013 /Revised: 19 August 2013 /Accepted: 10 September 2013 /Published online: 1 October 2013

(C) The Author(s) 2013. This article is published with open access at Springerlink.com

\begin{abstract}
The aim of this study was to investigate the association between signal transducer and activator of transcription 3 (STAT3) polymorphisms and autoimmune thyroid diseases and clinical features. We genotyped six single-nucleotide polymorphisms (SNPs) rs1053005, rs2293152, rs744166, rs17593222, rs2291281, and rs2291282 of STAT3 gene in 667 patients with autoimmune thyroid disease (417 Graves' disease (GD) and 250 Hashimoto's thyroiditis (HT)) and 301 healthy controls. The allele A from rs 1053005 was significantly less frequent in both GD and HT patients $(P=0.0024, \mathrm{OR}=0.6958,95 \% \mathrm{CI}=0.5508$ $0.8788 ; P=0.0091, \mathrm{OR}=0.7013,95 \% \mathrm{CI}=0.5397-0.9112$, respectively). The AA genotype of rs 1053005 was less in GD and HT patients too $(P=0.0025, \mathrm{OR}=0.6278,95 \% \mathrm{CI}=0.466$ $0.847)$ and $(P=0.0036, \mathrm{OR}=0.601,95 \% \mathrm{CI}=0.428-0.843)$. The allele $\mathrm{G}$ from rs17593222 increased the susceptibility to the ophthalmopathy development both in autoimmune thyroid disease (AITD) and GD patients $(P=0.0007, \mathrm{OR}=3.980,95 \% \mathrm{CI}=$ $1.871-8.464 ; P=0.0081, \mathrm{OR}=3.378,95 \% \mathrm{CI}=1.441-7.919$, respectively). The allele A and AA genotype of SNP rs1053005 may protect individuals from the susceptibility to AITD and their frequency decreased in AITD patients. In addition, the allele $\mathrm{G}$ of rs17593222 may increase the ophthalmopathy risk
\end{abstract}

\footnotetext{
L. Xiao $\cdot$ F.-S. Muhali $\cdot$ T.-t. Cai $\cdot$ R.-h. Song $\cdot$ X.-h. Shi $\cdot$

W.-j. Jiang • D.-F. Li $\cdot$ S.-t. He $\cdot$ J. Xu $\cdot$ J.-a. Zhang $(\triangle)$

Department of Endocrinology, Jinshan Hospital of Fudan

University, No. 1508 Longhang Road, Shanghai 201508,

People's Republic of China

e-mail: zhangjinan@hotmail.com

L. Xiao $\cdot$ R. Hu

Institute of endocrinology and Diabetology, Huashan Hospital

of Fudan University, No.12 Wulumuqizhong Road,

Shanghai 200040, People's Republic of China

F.-S. Muhali • T.-t. Cai $\cdot$ R.-h. Song

Department of Endocrinology, The First Affiliated Hospital of

Medical School of Xi' an Jiaotong University, No. 277 West Yanta

Road, Xi'an 710061, People's Republic of China
}

in AITD patients. Our findings suggest the existence of association between STAT3 gene and AITD, thus adding STAT3 gene to the list of the predisposing genes to AITD.

Keywords Signal transducer and activator of transcription 3 (STAT3) · Single-nucleotide polymorphisms (SNPs) ·

Autoimmune thyroid disease · Graves' disease · Hashimoto's thyroiditis

\section{Introduction}

Autoimmune thyroid diseases (AITDs), including Graves' disease (GD) and Hashimoto's thyroiditis (HT), are among the most common human autoimmune diseases. The prevalence in Caucasians is $1 \%$ (Hollowell et al. 2002). GD is characterized clinically by hyperthyroidism, diffuse goiter, and the presence of thyrotropin receptor antibodies, and HT is characterized by apoptosis of thyrocytes leading to hypothyroidism and the presence of thyroid peroxidase antibodies (TPOAb) or antibodies against thyroglobulin (TGAb). Despite their contrasting clinical presentations, GD and HT share many common features, mainly the infiltration of the thyroid by $\mathrm{T}$ cells and the production of anti-thyroid autoantibodies (Tomer 1997). The pathogenesis for AITDs remains unclear, though it is thought to involve several risk factors, including genetic risk factors (Tomer and Davies 2003) and environmental triggers such as cigarette smoking, iodine intake, and infection (Tomer and Davies 1993).

$\mathrm{T}$ helper cells are a group of immune cells that mediate adaptive immunity in vertebrates and are comprised of four major subtypes, Th1, Th2, Th17, and Treg (Dong 2008; Egwuagu 2009; Korn et al. 2009). In comparison to other T helper subsets, IL-17-producing T cells (Th17) are present in very low amounts in human blood but become highly elevated during chronic inflammation and are implicated in the pathology 
of several autoimmune diseases and chronic inflammatory disorders (Amadi-Obi et al. 2007). Recently, one of our studies identified the association of interleukin-17A and interleukin-17F gene single-nucleotide polymorphisms with autoimmune thyroid diseases. IL-17F/rs763780 polymorphisms may affect the susceptibility to AITD, and IL-17A/rs3819025 SNP is likely a protective factor to GD in the Chinese population (Yan et al. 2012). IL-17A and IL-17F are two IL-17 cytokine family members responsible for the pathogenic activity of the Th17 cells (Rutitzky et al. 2005). The differentiation of naive CD4+T cells toward the Th17 developmental pathway is mediated through activation of STAT3 pathways and Th17 lineage-specific transcription factors (Ivanov et al. 2007; Yang et al. 2007; Dong 2008; Korn et al. 2009). IL-6, IL-21, and IL-23 are all cytokines that activate the transcription factor STAT3 (Chen et al. 2007). Loss of STAT3 expression abrogates Th17 differentiation and inhibits the production of cytokines secreted by Th17 cells (Ivanov et al. 2007).

STAT3 gene polymorphisms had been reported to be associated with several immune diseases, including Crohn's disease (CD) (Ferguson et al. 2010), type1diabetes mellitus (Fung et al. 2009), Sjogren's syndrome (Okuma et al. 2013), and psoriatic arthritis (PsA) (Cenit et al. 2013). No reports about STAT3 SNPs' correlation to AITDs had been seen.

In this study, we attempted to find the association of SNPs rs1053005, rs2293152, rs744166, rs17593222, rs2291281, and rs2291282 at the STAT3 gene locus with GD and HT in a Han Chinese population.

\section{Materials and methods}

\section{Subjects}

All AITD (GD and HT) patients in the present case-control study were recruited from the Department of Endocrinology, the First Affiliated Hospital of Xi' an Jiaotong University. The diagnostic criteria for GD were mainly determined by clinical manifestation and laboratory biochemical proof of hyperthyroidism and the presence of diffuse goiter, the positive circulating TSH receptor antibody (TRAb) and antibody against thyroglobulin $(\mathrm{TgAb})$ or antibody against thyroid peroxidase (TPOAb). HT was defined on the basis of enlarged thyroid and the high level of either TPOAb or TgAb, with or without clinical and biochemical hypothyroidism. Fine needle aspiration was performed for any demonstrative and suspected cases of hypothyroidism. Three hundred and one healthy controls were recruited from unrelated physical examination individuals in the Health Check-up Center of the same hospital, with thyroid disease and other autoimmune diseases ruled out. All the subjects, including AITD patients and controls, were Han Chinese and signed the informed consent. The research project was approved by the Ethics Committee of the First
Affiliated Hospital of Medical School of Xi'an Jiaotong University. As shown in Table 1, our study investigated 667 AITD patients, who comprised 417 GD (124 men and 293 women) and $250 \mathrm{HT}$ patients (39 men and 211 women). In GD patients, the average onset age was $32.31 \pm 14.07,72$ individuals had family history and 98 had ophthalmopathy. In HT patients, the average onset age was $30.29 \pm 13.05,54$ individuals had family history and 6 had ophthalmopathy.

\section{Genotyping}

Peripheral venous blood of $2 \mathrm{ml}$ from the subjects was collected in an EDTA tube. The genomic DNA was extracted by saltingout method, using RelaxGene Blood DNA System (TIANGEN BIOTECH, Beijing, China), according to the manufactures' protocol. Genotyping of SNPs (rs1053005, rs2293152, rs744166, rs17593222, rs2291281, and rs2291282) was performed by MassARRAY system (Sequenom, San Diego, CA, USA) using the chip-based matrix-assisted laser desorption ionization time-of-flight mass spectrometry technology (Du et al. 2011). This is a well-selected platform by many researchers during studies including SNP genotyping. It has high specificity and sensitivity (Xiu-Cheng Fan et al. 2008). Primers were obtained from Sangon Biotech (Shanghai, China). Briefly, multiplex reaction was designed using Assay Designer software version 3.0 (Sequenom) and was processed following standard protocols for iPLEX chemistry. The reaction products were then cleaned and dispensed onto a SpectroCHIP bioarray. The chip was scanned using MassARRAY workstation version 3.3, and the resulting spectra were analyzed using the Sequenom TYPER software.

Table 1 Clinical data of AITD patients and controls

\begin{tabular}{llll}
\hline & GD & HT & Control \\
\hline $\begin{array}{l}\text { Number } \\
\text { Gender }\end{array}$ & 417 & 250 & 301 \\
$\quad$ Female & 293 & 211 & 210 \\
$\quad$ Male & 124 & 39 & 91 \\
Age & $34.48 \pm 13.95$ & $31.9 \pm 13.1$ & $33.5 \pm 12.59$ \\
Onset of age & $32.31 \pm 14.07$ & $30.29 \pm 13.05$ & \\
Thyroid size & & & \\
$\quad$ Normal & 85 & 41 & \\
I & 35 & 17 & \\
II & 231 & 169 & \\
III & 66 & 23 & \\
Family history & & 54 & \\
$(+)$ & 72 & 196 & \\
$(-)$ & 345 & 6 & \\
Ophthalmopathy & & 244 \\
$(+)$ & 98 & & \\
$(-)$ & 319 & &
\end{tabular}


Clinical phenotype analysis

Patients' clinical characteristics data were presented in Table 1. We conducted investigation in autoimmune thyroid patients (GD and HT) and their clinical features subphenotypes. Association analyses between genotypes and clinical manifestations of GD or HT were separately investigated involving (1) presence or absence of AITD family history (defined as the subjects' first-degree relatives including parents, children, and siblings or second-degree relatives such as grandparents, uncles, and aunts who had AITD occurrence); (2) presence or absence of ophthalmopathy (defined as a distinctive disorder characterized by inflammation and swelling of the extraocular muscles and orbital fat, eyelid retraction, periorbital edema, episcleral vascular injection, conjunctive swelling, and proptosis).

Statistical analysis

The clinical data are expressed as $M \pm \mathrm{SD}$. All SNPs were analyzed using HapLoView 4.2 software to perform Hardy-
Weinberg equilibrium (HWE) tests. Haplotype blocks were generated using the default algorithm based on methods established by Gabriel et al. (2002). Because all SNPs studied here were not located in the same block (data not shown), we did no haplotype frequency calculation and linkage disequilibrium (LD) test. Allele and genotype frequencies between cases and controls were computed by chi square test or Fisher's exact test. Differences between groups were determined by the odds ratio (OR) and $95 \%$ confidence interval $(95 \% \mathrm{CI})$. All statistical analyses were performed using the software GraphPad Prism 5. A $p$ value less than 0.05 was considered significant.

\section{Results}

Allele and genotype results

All of these six SNPs in both case and control groups were in HWE $(p>0.05)$. Table 2 shows the allele and

Table 2 Allele and genotype frequencies in AITD patients and controls

\begin{tabular}{|c|c|c|c|c|c|c|c|c|c|c|}
\hline SNP & Alleles & Con $(\%)$ & CD (\%) & $P$ & OR & $95 \% \mathrm{CI}$ & HT $(\%)$ & $P$ & OR & $95 \% \mathrm{CI}$ \\
\hline \multirow[t]{5}{*}{ rs 1053005} & G & $153(25.5)$ & $275(32.97)$ & 0.0024 & 0.6958 & $0.5508-0.8788$ & $164(32.8)$ & 0.0091 & 0.7013 & $0.5397-0.9112$ \\
\hline & A & 447 (74.5) & $559(67.03)$ & & & & $336(67.2)$ & & & \\
\hline & $\mathrm{AA}$ & $170(56.67)$ & $188(45.08)$ & 0.008 & & & $110(44)$ & 0.0125 & & \\
\hline & $\mathrm{AG}$ & $107(35.67)$ & $183(43.88)$ & & & & $116(46.4)$ & & & \\
\hline & GG & $23(7.66)$ & $46(11.04)$ & & & & $24(9.6)$ & & & \\
\hline \multirow[t]{4}{*}{ rs17593222 } & $\mathrm{C}$ & $586(97.67)$ & $810(97.36)$ & 0.7363 & & & 493 (98.6) & 0.279 & & \\
\hline & G & $14(2.33)$ & $22(2.64)$ & & & & $7(1.4)$ & & & \\
\hline & $\mathrm{CC}$ & $286(95.33)$ & 394 (94.71) & 0.7329 & & & $243(97.2)$ & 0.2744 & & \\
\hline & $\mathrm{CG}$ & $14(4.67)$ & $22(5.29)$ & & & & $7(2.8)$ & & & \\
\hline \multirow[t]{4}{*}{ rs2291281 } & A & $8(1.33)$ & $6(0.72)$ & 0.2821 & & & $6(1.2)$ & & & \\
\hline & G & $592(98.67)$ & $828(99.28)$ & & & & $492(98.8)$ & & & \\
\hline & $\mathrm{AG}$ & $8(2.67)$ & $6(1.44)$ & 0.2799 & & & $6(2.41)$ & & & \\
\hline & GG & $292(97.33)$ & $411(98.56)$ & & & & $243(97.59)$ & & & \\
\hline \multirow[t]{4}{*}{ rs2291282 } & $\mathrm{C}$ & 7 (1.17) & $17(2.04)$ & 0.2194 & & & $4(0.8)$ & 0.7627 & & \\
\hline & $\mathrm{T}$ & $593(98.83)$ & $817(97.96)$ & & & & $496(99.2)$ & & & \\
\hline & $\mathrm{CT}$ & $7(2.33)$ & $17(4.08)$ & 0.2156 & & & $4(1.6)$ & 0.7615 & & \\
\hline & $\mathrm{TT}$ & $293(97.67)$ & $400(95.92)$ & & & & $246(98.4)$ & & & \\
\hline \multirow[t]{5}{*}{ rs2293152 } & $\mathrm{C}$ & $293(49)$ & 371 (44.7) & 0.1189 & & & 234 (46.99) & 0.5438 & & \\
\hline & G & $305(51)$ & $459(55.3)$ & & & & $264(53.01)$ & & & \\
\hline & $\mathrm{CC}$ & $68(22.74)$ & $79(19.04)$ & 0.2577 & & & 53 (21.29) & 0.7764 & & \\
\hline & $\mathrm{CG}$ & $157(52.51)$ & $213(51.33)$ & & & & $128(51.41)$ & & & \\
\hline & 66 & $74(24.75)$ & $123(29.63)$ & & & & $68(27.3)$ & & & \\
\hline \multirow[t]{5}{*}{ rs744166 } & $\mathrm{C}$ & $203(34.06)$ & $161(38.15)$ & 0.1849 & & & 199 (39.8) & 0.0513 & & \\
\hline & $\mathrm{T}$ & $393(65.94)$ & $261(61.85)$ & & & & $301(60.2)$ & & & \\
\hline & $\mathrm{CC}$ & $34(11.41)$ & $22(10.43)$ & 0.0704 & & & $41(16.4)$ & 0.1381 & & \\
\hline & $\mathrm{CT}$ & $135(45.3)$ & $117(55.45)$ & & & & $117(46.8)$ & & & \\
\hline & $\mathrm{TT}$ & $129(43.29)$ & $72(34.12)$ & & & & $92(36.8)$ & & & \\
\hline
\end{tabular}


Table 3 Genotype distributions of rs1053005 and rs744166 in AITD patients and controls

\begin{tabular}{|c|c|c|c|c|c|c|c|c|c|c|}
\hline SNP & Genotype & Con $(\%)$ & GD (\%) & $P$ & OR & $\mathrm{CI}$ & HT (\%) & $P$ & OR & CI \\
\hline \multirow[t]{2}{*}{ rs1053005 } & $\mathrm{AG}+\mathrm{GG}$ & $130(43.33)$ & $229(54.92)$ & 0.0025 & 0.6278 & $0.466-0.847$ & $140(56)$ & 0.0036 & 0.601 & $0.428-0.843$ \\
\hline & $\mathrm{AA}$ & $170(56.67)$ & $188(45.08)$ & & & & $110(44)$ & & & \\
\hline \multirow[t]{2}{*}{ rs744166 } & $\mathrm{CT}+\mathrm{CC}$ & $169(56.71)$ & $139(65.88)$ & 0.0429 & 0.6786 & $0.471-0.978$ & $158(63.2)$ & 0.1374 & & \\
\hline & TT & $129(43.29)$ & $72(34.12)$ & & & & $92(36.8)$ & & & \\
\hline
\end{tabular}

genotype frequencies and case-control association analysis for each SNP. In rs1053005, we found that allele A was significantly less frequent in GD and HT patients than in controls $(p=0.0024, \mathrm{OR}=0.6958,95 \% \mathrm{CI}=$ $0.5508-0.8788)$ and $(p=0.0091, \mathrm{OR}=0.7013,95 \% \mathrm{CI}=$ $0.5397-0.9112$ ) (shown in Table 2). Also, AA genotype of rs1053005 was less in GD ( $p=0.0025, \mathrm{OR}=0.6278$, $95 \% \mathrm{CI}=0.466-0.847)$ and $\mathrm{HT}(p=0.0036, \mathrm{OR}=0.601$, $95 \% \mathrm{CI}=0.428-0.843)$ patients than the control, which evidently indicated the AA genotype could decrease the susceptibility to GD and HT (shown in Table 3). SNP rs744166 showed a marginal significant trend between HT subjects and control group ( $p=0.0513)$ (shown in Table 2), while the distribution of TT genotype from rs744166 ( $p=$ $0.0429, \mathrm{OR}=0.6786,95 \% \mathrm{CI}=0.471-0.978$ ) was decreased in GD patients compared to the healthy controls (shown in Table 3). Nevertheless, we did not find any significant difference both in genotype and allele frequencies between patients and controls either in rs17593222, rs2291281, rs2291282, or in rs2293152 SNPs.
Genotype and clinical phenotype associations

When we further compared the SNP rs1053005 between patients with and those without family history, in both GD and HT patients, no significant difference was observed (data not shown).

Four SNPs, including rs1053005, rs17593222, rs2293152, and rs744166, showed a significant association when ophthalmopathy AITD patients were compared to the healthy controls (shown in Table 4). Only two SNPs (rs1053005 and rs744166) in non-ophthalmopathy AITD patients showed difference when compared to the controls (shown in Table 4). When we compared the ophthalmopathy to the non-ophthalmopathy within AITD patients, SNP rs17593222 showed significant association $(p=0.0007, \mathrm{OR}=3.980,95 \% \mathrm{CI}=1.871-8.464)$.

Graves' ophthalmopathy (GO) also known as thyroidassociated ophthalmopathy is a condition that primarily affects the extraocular muscles, the muscles that rotate the eyeball up, down, and to the sides. It can happen

Table 4 The allele and genotype frequencies of rs1053005, rs17953222, rs2293152, and rs744166 in ophthalmopathy and non-ophthalmopathy patients and controls

\begin{tabular}{|c|c|c|c|c|c|c|c|c|c|c|}
\hline SNP & Alleles & Con $(\%)$ & Ophthalmopathy & $P$ & OR & CI & Non-ophthalmopathy & $P$ & OR & $\mathrm{CI}$ \\
\hline \multirow[t]{4}{*}{ rs1053005 } & A & $447(74.5)$ & $140(67.31)$ & 0.0475 & 1.419 & $1.007-2.000$ & $753(66.99)$ & 0.0012 & 1.439 & $1.153-1.797$ \\
\hline & G & $153(25.5)$ & $68(32.69)$ & & & & $371(33.01)$ & & & \\
\hline & $\mathrm{AA}$ & $170(56.67)$ & $48(46.15)$ & 0.0685 & & & $249(44.31)$ & 0.0006 & 1.644 & $1.239-2.181$ \\
\hline & $\mathrm{AG}+\mathrm{GG}$ & $130(43.33)$ & $56(53.85)$ & & & & $313(55.69)$ & & & \\
\hline \multirow[t]{4}{*}{ rs17593222 } & $\mathrm{C}$ & $586(97.67)$ & $196(94.23)$ & 0.0218 & 2.563 & $1.165-5.635$ & $1105(98.48)$ & 0.2545 & & \\
\hline & G & $14(2.33)$ & $12(5.77)$ & & & & $17(1.52)$ & & & \\
\hline & $\mathrm{CC}$ & $286(95.33)$ & $92(88.46)$ & 0.0197 & 2.665 & $190-5.967$ & $544(96.97)$ & 0.2502 & & \\
\hline & CG & $14(4.67)$ & $12(11.54)$ & & & & $17(3.03)$ & & & \\
\hline \multirow[t]{4}{*}{ rs 2293152} & $\mathrm{C}$ & $293(49)$ & $79(37.98)$ & 0.0061 & 1.569 & $1.136-2.166$ & $521(46.60)$ & 0.3612 & & \\
\hline & G & $305(51)$ & $129(62.02)$ & & & & $597(53.40)$ & & & \\
\hline & GG & $74(24.75)$ & $44(42.31)$ & 0.0011 & 0.4485 & $0.280-0.717$ & $150(26.83)$ & 0.5681 & & \\
\hline & $\mathrm{CG}+\mathrm{CC}$ & $225(75.25)$ & $60(57.69)$ & & & & 409 (73.17) & & & \\
\hline \multirow[t]{4}{*}{ rs 744166} & $\mathrm{C}$ & $203(34.06)$ & $87(42.23)$ & 0.0433 & 0.7065 & $0.511-0.977$ & $444(39.57)$ & 0.0280 & 0.7888 & $0.641-0.971$ \\
\hline & $\mathrm{T}$ & $393(65.94)$ & 119 (57.77) & & & & $678(60.43)$ & & & \\
\hline & $\mathrm{CC}$ & $34(11.41)$ & $20(19.42)$ & 0.0454 & 0.5345 & $0.292-0.979$ & $87(15.51)$ & 0.7017 & & \\
\hline & $\mathrm{CT}+\mathrm{TT}$ & $264(88.59)$ & $83(80.58)$ & & & & $474(84.49)$ & & & \\
\hline
\end{tabular}


Table 5 Ophthalmopathy in ATD disease

\begin{tabular}{llllll}
\hline & Ophthalmopathy (\%) & Non-ophthalmopathy (\%) & $P$ & OR & CI \\
\hline GD & $98(94.23)$ & $319(56.76)$ & $<0.0001$ & 12.44 & $5.365-28.86$ \\
HT & $6(5.77)$ & $243(43.24)$ & & & \\
\hline
\end{tabular}

not only in GD but also in HT patients. Nevertheless, we can see GD contributed much more to the development of ophthalmopathy as shown in the Table 5.

Allele $G$ from rs17593222 also increased the risk of ophthalmopathy $(p=0.0081, \mathrm{OR}=3.378,95 \% \mathrm{CI}=1.441-$ 7.919) within GD patients. The minor allele $\mathrm{G}$ from rs17593222 increased the susceptibility to the ophthalmopathy development both in AITD and GD patient subgroups (shown in Tables 6 and 7). CG genotype of $\mathrm{rs}$ 17593222 SNP increased the risk of ophthalmopathy too when compared to $\mathrm{CC}$ genotype $(p=0.0073, \mathrm{OR}=$ $3.529,95 \% \mathrm{CI}=1.480-8.416)$. GG genotype from SNP rs2293152 also increased the risk of ophthalmopathy in GD patients $(p=0.031, \mathrm{OR}=1.729,95 \% \mathrm{CI}=1.073-$ 2.784) (shown in Table 7). No differences were seen in other SNPs, including rs1053005 and rs744166 (data not shown).

\section{Discussion}

STAT3 gene is located on chromosome17q21.31. The protein encoded by this gene is a member of the STAT protein family. In response to cytokines and growth factors, STAT family members are phosphorylated by the receptor-associated kinases and then form homo- or heterodimers that translocate to the cell nucleus where they act as transcription activators (Zhong et al. 1994). This protein is activated through phosphorylation in response to various cytokines and growth factors including IFNs, EGF, IL5, IL6, HGF, LIF, and BMP2. It mediates the expression of many genes in response to cell stimuli and thus plays a key role in many cellular processes such as cell growth and apoptosis. STAT3 also plays key roles in Th17 lymphocyte development and differentiation.

SNPs rs744166, rs2293152, and rs1053005 had been found to be associated with many autoimmune diseases. The association with psoriatic arthritis (PsA) in SNP rs744166 was observed in Spanish Caucasian origin (Cenit et al. 2013). So did its association with ankylosing spondylitis (AS) in white European ancestry (Danoy et al. 2010) and that with multiple sclerosis (MS) in Finland population (Jakkula et al. 2010). The susceptibility of STAT3 rs744166 TT homozygotes for ulcerative colitis (UC) was found in Hungarian (Polgar et al. 2012). Also, the association of rs744166 to Crohn's disease (CD) was also confirmed in adult German (Prager et al. 2012). STAT3 (rs2293152, rs1053005) were significantly associated with AS in Han Chinese (Davidson et al. 2011). A significantly increased frequency of the GG genotype of the STAT3 rs2293152 was also observed in patients with $\mathrm{BD}$ in the Han Chinese population (Hu et al. 2012). The frequencies of a $\mathrm{C}$ allele and its homozygous CC genotype at rs2293152 SNP in STAT3 in CD patients were significantly higher than those in control subjects in the Japanese population (Sato et al. 2009). However, no study about the STAT3 SNPs association with AITDs was reported until now.

Our study examined the association between six SNPs of STAT3 gene and AITD in a Chinese Han population. Regarding the allele frequencies and genotype analysis of STAT3, we found that rs1053005 major allele A was significantly decreased in GD and HT patients and lowered the risk

Table 6 The allele and genotype frequencies of rs17593222 and rs2293152 in ophthalmopathy and non-ophthalmopathy AITD patients

\begin{tabular}{|c|c|c|c|c|c|c|}
\hline SNP & Alleles & Non-ophthalmopathy (\%) & Ophthalmopathy (\%) & $P$ & OR & CI \\
\hline \multirow[t]{4}{*}{ rs17593222 } & $\mathrm{C}$ & $1105(98.48)$ & $196(94.23)$ & 0.0007 & 3.980 & $1.871-8.464$ \\
\hline & $\mathrm{G}$ & $17(1.52)$ & $12(5.77)$ & & & \\
\hline & $\mathrm{CC}$ & $544(96.97)$ & $92(88.46)$ & 0.0006 & 4.174 & $1.930-9.028$ \\
\hline & CG & $17(3.03)$ & $12(11.54)$ & & & \\
\hline \multirow[t]{4}{*}{ rs2293152 } & $\mathrm{C}$ & $521(46.60)$ & $82(39.42)$ & 0.0583 & & \\
\hline & $\mathrm{G}$ & $597(53.40)$ & $126(60.58)$ & & & \\
\hline & $\mathrm{CG}+\mathrm{CC}$ & $409(73.17)$ & $63(60.58)$ & 0.0129 & 1.774 & $1.148-2.743$ \\
\hline & GG & $150(26.83)$ & $41(39.42)$ & & & \\
\hline
\end{tabular}


Table 7 The allele and genotype frequencies of rs1759222 and rs2293152 in ophthalmopathy and non-ophthalmopathy GD patients

\begin{tabular}{|c|c|c|c|c|c|c|}
\hline SNP & Alleles & Non-ophthalmopathy GD (\%) & Ophthalmopathy GD (\%) & $P$ & OR & $\mathrm{CI}$ \\
\hline \multirow[t]{4}{*}{ rs17593222 } & $\mathrm{C}$ & $625(98.27)$ & $185(94.39)$ & 0.0081 & 3.378 & $1.441-7.919$ \\
\hline & G & $11(1.73)$ & $11(5.61)$ & & & \\
\hline & $\mathrm{CC}$ & $307(96.54)$ & 87 (88.78) & 0.0073 & 3.529 & $1.480-8.416$ \\
\hline & $\mathrm{CG}$ & $11(3.46)$ & $11(11.22)$ & & & \\
\hline \multirow[t]{4}{*}{ rs2293152 } & $\mathrm{C}$ & $293(46.21)$ & $78(39.80)$ & 0.1190 & & \\
\hline & G & $341(53.79)$ & $118(60.20)$ & & & \\
\hline & $\mathrm{CG}+\mathrm{CC}$ & $232(73.19)$ & $60(61.22)$ & 0.031 & 1.729 & $1.073-2.784$ \\
\hline & GG & $85(26.81)$ & $38(38.78)$ & & & \\
\hline
\end{tabular}

of diseases by 30.42 and $29.87 \%$, respectively. The AA genotype frequencies in control, GD, and HT collections were $56.67,45.08$, and $44 \%$, respectively. There is a clear observation that the AA could lower the risk of GD or HT by 37.22 and $39.9 \%$, respectively, and protect individuals from developing AITDs. SNP rs744166 showed a marginal difference between the HT and control groups $(p=0.0513)$. We observed much higher risk of ophthalmopathy development both in AITD and GD group with G allele of rs 17593222 $(\mathrm{OR}=3.980$ and 3.378 , respectively).

Dysregulation of effector $\mathrm{T}$ cell responses represents a common feature of a wide range of autoimmune diseases. Activated CD4+ T cells differentiate into distinct functional subsets, characterized by heritable patterns of cytokine secretion and the expression of specific transcription factors. IL-17 is mostly known for its pro-inflammatory activities, both in vitro and in vivo, and its expression is increased in inflammatory tissues (Kolls and Linden 2004). One of these subsets, CD4+ T cells that preferentially produce IL-17, designed Th17, have attracted tremendous attention because of the connection with autoimmune disease (Stockinger and Veldhoen 2007; Weaver et al. 2007; Miossec et al. 2009). An increasing body of evidence shows the requirement of STAT3 for Th17 cell development. Patients suffering from autosomal dominant hyperIgE syndrome, associated with negative mutations in STAT3, had impaired Th17 cell differentiation (de Beaucoudrey et al. 2008; Ma et al. 2008; Milner et al. 2008; Renner et al. 2008).

STAT3 bound to and regulated multiple genes that contribute to the Th17 phenotype, including not only the IL17 locus itself, IL21, and IL23r but also CCR6 (Durant et al. 2010; Ghoreschi et al. 2010; Yang et al. 2011). STAT3 also bound to genes encoding transcription factors critical for Th17 differentiation including Rorc (which encodes Ror $\gamma \mathrm{t}$ ), Irf4, Batf, and Nfibiz (Durant et al. 2010).

We and other researchers have found that there is an increased differentiation of Th17 lymphocytes and an enhanced synthesis of Th17 cytokines in AITDs. These may substantially contribute to the pathogenesis of thyroid autoimmunity (Figueroa-Vega et al. 2010; Qin et al. 2012). Our previous work had also found the association of IL-17A and IL-17F SNPs with AITDs, which were two IL-17 cytokine family members secreted by Th17 cells (Yan et al. 2012).

Immunohistochemical analysis had demonstrated differential expression patterns of the various members of the STAT transcription factors in lymphocytic thyroiditis, indicating that each member of this conserved protein family has its distinct functions in the development of the disease. The cell-type-specific expression patterns of STAT proteins in human lymphocytic thyroiditis reflect their distinct and partially antagonistic roles in orchestrating the balance between degenerating and regenerating processes within a changing cytokine environment (Staab et al. 2012). STAT protein members have different effect on Th17 differentiation and may contribute to their roles in human lymphocytic thyroiditis pathogenesis.

STAT3 is a key signaling molecule within the Th17 lymphocyte differentiation pathway. It may play roles in AITDs and ophthalmopathy development by regulating Th17 lymphocyte differentiation. Here, we report the contribution of STAT3 SNPs to AITDs and ophthalmopathy susceptibility in patients for the first time. The AITDs occurrence may be attributed to altered differentiation of Th17 lymphocytes and different synthesis of Th17 cytokines in the tissue.

Acknowledgments This project was supported by grants from the National Natural Science Foundation of China (30871184, 81070627).

Conflict of interest The authors declare no competing interest. The authors alone are responsible for the content and writing of the paper.

Open Access This article is distributed under the terms of the Creative Commons Attribution License which permits any use, distribution, and reproduction in any medium, provided the original author(s) and the source are credited. 


\section{References}

Amadi-Obi A, Yu CR et al (2007) TH17 cells contribute to uveitis and scleritis and are expanded by IL-2 and inhibited by IL-27/STAT1. Nat Med 13(6):711-718

Cenit MC, Ortego-Centeno N et al (2013) Influence of the STAT3 genetic variants in the susceptibility to psoriatic arthritis and Behcet's disease. Hum Immunol 74(2):230-233

Chen Z, Laurence A et al (2007) Signal transduction pathways and transcriptional regulation in the control of Th17 differentiation. Semin Immunol 19(6):400-408

Danoy P, Pryce K et al (2010) Association of variants at 1q32 and STAT3 with ankylosing spondylitis suggests genetic overlap with Crohn's disease. PLoS Genet 6(12):e1001195

Davidson SI, Liu Y et al (2011) Association of STAT3 and TNFRSF1A with ankylosing spondylitis in Han Chinese. Ann Rheum Dis 70(2): 289-292

de Beaucoudrey L, Puel A et al (2008) Mutations in STAT3 and IL12RB1 impair the development of human IL-17-producing T cells. J Exp Med 205(7):1543-1550

Dong C (2008) TH17 cells in development: an updated view of their molecular identity and genetic programming. Nat Rev Immunol 8(5):337-348

Du J, Huo J et al (2011) Polymorphisms of nuclear factor-kappaB family genes are associated with development of multiple myeloma and treatment outcome in patients receiving bortezomibbased regimens. Haematologica 96(5):729-737

Durant L, Watford WT et al (2010) Diverse targets of the transcription factor STAT3 contribute to T cell pathogenicity and homeostasis. Immunity 32(5):605-615

Egwuagu CE (2009) STAT3 in CD4+ T helper cell differentiation and inflammatory diseases. Cytokine 47(3):149-156

Ferguson LR, Han DY et al (2010) Genetic factors in chronic inflammation: single nucleotide polymorphisms in the STATJAK pathway, susceptibility to DNA damage and Crohn's disease in a New Zealand population. Mutat Res 690(1-2): $108-115$

Figueroa-Vega N, Alfonso-Perez M et al (2010) Increased circulating pro-inflammatory cytokines and Th17 lymphocytes in Hashimoto's thyroiditis. J Clin Endocrinol Metab 95(2):953962

Fung EY, Smyth DJ et al (2009) Analysis of 17 autoimmune diseaseassociated variants in type 1 diabetes identifies 6q23/TNFAIP3 as a susceptibility locus. Genes Immun 10(2):188-191

Gabriel SB, Schaffner SF et al (2002) The structure of haplotype blocks in the human genome. Science 296(5576):2225-2229

Ghoreschi K, Laurence A et al (2010) Generation of pathogenic $\mathrm{T}(\mathrm{H}) 17$ cells in the absence of TGF-beta signalling. Nature 467(7318):967-971

Hollowell JG, Staehling NW et al (2002) Serum TSH, T(4), and thyroid antibodies in the United States population (1988 to 1994): National Health and Nutrition Examination Survey (NHANES III). J Clin Endocrinol Metab 87(2):489-499

Hu K, Hou S et al (2012) JAK2 and STAT3 polymorphisms in a Han Chinese population with Behcet's disease. Invest Ophthalmol Vis Sci 53(1):538-541

Ivanov II, Zhou L et al (2007) Transcriptional regulation of Th17 cell differentiation. Semin Immunol 19(6):409-417

Jakkula E, Leppa V et al (2010) Genome-wide association study in a high-risk isolate for multiple sclerosis reveals associated variants in STAT3 gene. Am J Hum Genet 86(2):285-291

Kolls JK, Linden A (2004) Interleukin-17 family members and inflammation. Immunity 21(4):467-476

Korn T, Bettelli E et al (2009) IL-17 and Th17 Cells. Annu Rev Immunol 27:485-517
Ma CS, Chew GY et al (2008) Deficiency of Th17 cells in hyper IgE syndrome due to mutations in STAT3. J Exp Med 205(7):1551-1557

Milner JD, Brenchley JM et al (2008) Impaired T(H)17 cell differentiation in subjects with autosomal dominant hyper-IgE syndrome. Nature 452(7188):773-776

Miossec P, Korn T et al (2009) Interleukin-17 and type 17 helper T cells. N Engl J Med 361(9):888-898

Okuma A, Hoshino K et al (2013) Enhanced apoptosis by disruption of the STAT3-IkappaB-zeta signaling pathway in epithelial cells induces Sjogren's syndrome-like autoimmune disease. Immunity 38(3):450-460

Polgar N, Csongei V et al (2012) Investigation of JAK2, STAT3 and CCR6 polymorphisms and their gene-gene interactions in inflammatory bowel disease. Int J Immunogenet 39(3):247-252

Prager M, Buttner J et al (2012) The JAK2 variant rs10758669 in Crohn's disease: altering the intestinal barrier as one mechanism of action. Int J Colorectal Dis 27(5):565-573

Qin Q, Liu P et al (2012) The increased but non-predominant expression of Th17- and Th1-specific cytokines in Hashimoto's thyroiditis but not in Graves' disease. Braz J Med Biol Res 45(12):1202-1208

Renner ED, Rylaarsdam S et al (2008) Novel signal transducer and activator of transcription 3 (STAT3) mutations, reduced $\mathrm{T}(\mathrm{H}) 17$ cell numbers, and variably defective STAT3 phosphorylation in hyper-IgE syndrome. J Allergy Clin Immunol 122(1):181-187

Rutitzky LI, Lopes da Rosa JR et al (2005) Severe CD4 T cellmediated immunopathology in murine schistosomiasis is dependent on IL-12p40 and correlates with high levels of IL-17. J Immunol 175(6):3920-3926

Sato K, Shiota M et al (2009) Strong evidence of a combination polymorphism of the tyrosine kinase 2 gene and the signal transducer and activator of transcription 3 gene as a DNAbased biomarker for susceptibility to Crohn's disease in the Japanese population. J Clin Immunol 29(6):815-825

Staab J, Barth PJ et al (2012) Cell-type-specific expression of STAT transcription factors in tissue samples from patients with lymphocytic thyroiditis. Endocr Pathol 23(3):141-150

Stockinger B, Veldhoen M (2007) Differentiation and function of Th17 T cells. Curr Opin Immunol 19(3):281-286

Tomer Y (1997) Anti-thyroglobulin autoantibodies in autoimmune thyroid diseases: cross-reactive or pathogenic? Clin Immunol Immunopathol 82(1):3-11

Tomer Y, Davies TF (1993) Infection, thyroid disease, and autoimmunity. Endocr Rev 14(1):107-120

Tomer Y, Davies TF (2003) Searching for the autoimmune thyroid disease susceptibility genes: from gene mapping to gene function. Endocr Rev 24(5):694-717

Weaver CT, Hatton RD et al (2007) IL-17 family cytokines and the expanding diversity of effector $\mathrm{T}$ cell lineages. Annu Rev Immunol 25:821-852

Xiu-Cheng Fan A, Garritsen HS et al (2008) A rapid and accurate approach to identify single nucleotide polymorphisms of mitochondrial DNA using MALDI-TOF mass spectrometry. Clin Chem Lab Med 46(3):299-305

Yan N, Yu YL et al (2012) Association of interleukin-17A and $17 \mathrm{~F}$ gene single-nucleotide polymorphisms with autoimmune thyroid diseases. Autoimmunity 45(7):533-539

Yang XO, Panopoulos AD et al (2007) STAT3 regulates cytokinemediated generation of inflammatory helper T cells. J Biol Chem 282(13):9358-9363

Yang XP, Ghoreschi K et al (2011) Opposing regulation of the locus encoding IL-17 through direct, reciprocal actions of STAT3 and STAT5. Nat Immunol 12(3):247-254

Zhong Z, Wen Z et al (1994) Stat3: A STAT family member activated by tyrosine phosphorylation in response to epidermal growth factor and interleukin-6. Science 264(5155):95-98 